\title{
Corneal Cross-linking in Combination with Intracorneal Ring Segments
}

Aylin Kılıç

\section{ABSTRACT}

The goal of ring segment surgery is to reduce the degree of myopia and astigmatism, improving uncorrected and corrected distance visual acuity. The crosslinking is a procedure used for stabilizing the cornea in patients with progressive keratoconus. Corneal crosslinking surgery and combination of an intracorneal ring segments seems to be as effective for corneal stability and refractive improvement in keratoconic eyes.

Keywords: Corneal cross-linking, Intracorneal ring segments, Keratoconus.

How to cite this article: Kılıç A. Corneal Cross-linking in Combination with Intracorneal Ring Segments. Int J Kerat Ect Cor Dis 2017;6(2):92-96.

\section{Source of support: Nil}

\section{Conflict of interest: None}

\section{INTRODUCTION}

The changes in corneal structure induced by additive technologies can be roughly predicted by the Barraquer "thickness law". When you add material to the periphery of the cornea or remove an equal amount of material from the central area, you achieve a resultant flattening effect. On the contrary, when you add material to the center or remove it from the corneal periphery, you obtain a steeped surface curvature. The corrective result varies in direct proportion to the thickness of the implant and is in inverse proportion to its diameter. The thicker and the smaller the device, the higher is the corrective result obtained.

According to the postulates of Barraquer and Blavatskaya, intracorneal ring acts as tissue addition leading to a flattening in the cornea periphery. ${ }^{1}$ The diameter of the ring is proportionally inverse to the flattening intensity. Thus, the smaller the diameter, the more tissue added (ring thickness) to the higher myopic correction.

\section{Assistant Professor}

Department of Ophthalmology, Medipol Mega University Hospital, İstanbul, Turkey

Corresponding Author: Aylin Kılıç, Assistant Professor Department of Ophthalmology, Medipol Mega University Hospital, İstanbul Turkey. e-mail: aylinkilicdr@gmail.com

\section{INTRACORNEAL RING SEGMENTS FOR KERATOCONUS}

Colin et al first described intracorneal ring segments (ICRS) as a treatment modality for keratoconus in $2001 .^{2}$ This study showed flattening of the cornea (reduction of the maximum K-value $>4.00 \mathrm{D}$ ) and reduction in spherical equivalent (SE) (>2.00 D), and improved best spectacle-corrected visual acuity (BCVA) (1 line) and uncorrected visual acuity (UCVA) (2 lines) in 10 patients with keratoconus. Since then, other investigators demonstrated similar outcome using a varying combination of segment sizes and number in a diverse group of patients. ${ }^{3-5}$ The collagen cross-linking (CXL) treatment introduced by Wollensak et $\mathrm{al}^{6}$ in 2003 was accompanied by a reduction of a lesser degree compared to ICRS of maximal keratometry (2.01 D) and refractive error $(1.14 \mathrm{D})$. It also increased corneal rigidity by 4.5 times and seemed to be an effective mean for stabilizing the cornea. ${ }^{7}$

In keratoconus, the corneal elastic modulus is reduced due to pathology in the corneal stroma. From a biomechanical perspective, the resistance to deformation is reduced in relation to the reduction of the elastic modulus that leads to increased strain and protrusion in the cornea (Fig. 1). The consequence is increased curvature and corneal thinning - the hallmarks of keratoconus. Since stress is defined as applied force divided by cross-sectional area, stress focally increases in the zone of corneal thinning. The

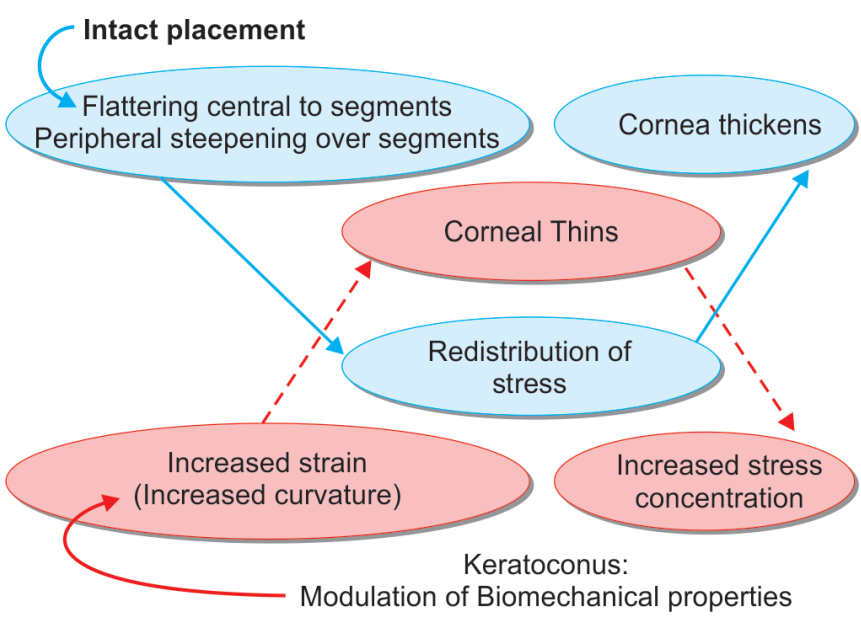

Fig. 1: Redistribution of corneal curvature leads to a redistribution of corneal stress, interrupting the biomechanical cycle of keratoconus disease progression and in some cases, reversing the process 
placement of ICRS generates both an immediate response that interrupts the biomechanical disease progression in keratoconus, and a time-dependent biomechanical response that allows subsequent improvement of vision over 6 months. The immediate response governed by the elastic properties and the long-term response is by viscoelastic properties. Intracorneal ring placement results in a reduction of astigmatism and improves visual acuity. This is accomplished by shortening the path length of the portion of the collagen lamellae, which are central to the segments. Redistribution of corneal curvature leads to a redistribution of corneal stress, interrupting the biomechanical cycle of keratoconus disease progression, and in some cases, reversing the process.

The CXL and intracorneal rings have theoretical complementary effects on corneal biomechanics and refractive parameters. The CXL has an effect mainly on the anterior cornea, and ICRS provide flattening effects and redistribution of stress on deeper layers.

\section{COLLAGEN CROSS-LINKING AND ICRS FOR KERATOCONUS}

The CXL is a procedure used for stabilizing the cornea in patients with progressive keratoconus by increasing corneal rigidity, but without major refractive and visual changes. The ICRS act by flattening the center of the cornea. Originally designed for the correction of mild myopia in normal eyes, the segments are now commonly used to regularize the corneal surface in keratoconus to improve UCVA and CVA and improve the ability to wear soft contact lenses. Although the ICRS mechanism is not totally understood, segment insertion induces a change in corneal volume peripherally and overall corneal curvature, with possible modification of corneal biomechanical properties. Combining ICRS with CXL could be synergistic in terms of biomechanical efficacy, and thus a valuable treatment for progressive keratoconus (KC) progression in addition to the potential for improved acuity for ICRS placement.

The goal of ring segment surgery is to reduce the degree of myopia and astigmatism, improving uncorrected distance visual acuity (UDVA) and corrected distance visual acuity (CDVA). Shifting the position of the cone more centrally within the cornea may also improve higher-order aberrations, such as coma. The primary goal of ICRS is to decrease the very high refractive errors to a refractive level allowing a corrected visual acuity compatible with a satisfactory quality of life. Therefore, this minimally invasive treatment found a place of choice in the surgical management of keratoconic patient by avoiding more invasive surgical procedures, such as keratoplasty. This goal is usually achieved approximately $70 \%$ of the time.

\section{COLLAGEN CROSS-LINKING AND ICRS: SEQUENTIAL VS SIMULTANEOUS TREATMENTS}

Sequential or simultaneous treatments with both intracorneal rings and CXL have been evaluated in short series of patients, and it seems that a synergistic effect, compared to either procedure alone, has emerged from these studies. Combining both procedures should result in greater improvement of UCVA and BCVA, a greater reduction of myopia and astigmatism, and more than twofold reduction of the mean and the steepest keratometry readings compared to either procedure alone. Postulated explanations for the greater effects include: A simple addition of the effect of the two procedures, coupling from cross-linking of collagen around the ring segments, and greater effect of cross-linking due to pooling and concentration of riboflavin in the channel around the ring segments. Simultaneous implantation of corneal rings and CXL could provide the advantage of delivering riboflavin to the cornea by direct injection into the stromal channel, avoiding the need for epithelial removal.

Chan et $\mathrm{al}^{8}$ were the first to compare the combined treatment of Intacs and CXL with the treatment of Intacs implantation alone. The CXL was performed immediately after Intacs implantation. They reported a significant reduction in cylinder (2.73 vs $1.48 \mathrm{D})$, steepest keratometry (1.94 vs $0.89 \mathrm{D})$, and Colin et al reported that with CXL in combination with intrastromal rings average keratometry reduced (1.34 vs $0.21 \mathrm{D})$ after the combined treatment compared to the ICRS treatment alone. Ertan et $\mathrm{al}^{9}$ reported an additional improvement in UCVA (1.9 lines), BCVA (1.7 lines), SE (2.8 D), cylinder (0.47 D), and mean $\mathrm{K}$ after CXL in patients who had already been implanted with Intacs rings, on average 7 months before. The authors have, therefore, suggested that CXL had an additive effect on Intacs implantation and might be considered an enhancement/stabilizing procedure. More recently, a prospective and randomized study conducted by Coskunseven has compared the two sequences of CXL followed by ICRS implantation or ICRS implantation followed by CXL. ${ }^{10}$ The mean interval between the procedures was 7 months. They reported that the first sequence (ICRS implantation followed by CXL) led to a greater improvement in BCVA (3 lines vs 1 line), SE (3.31 vs $2.76 \mathrm{D})$ and manifest cylinder (2.08 vs $1.32 \mathrm{D})$ than patients who received CXL first. The authors suggested that the corneal stiffness induced by CXL might reduce the flattening effect of ICRS. Therefore, they concluded that the CXL and ICRS combination had a greater effect than ICRS alone, regardless of the sequence used.

However, some studies have not found synergistic effects of combining both treatments. Renesto et $\mathrm{al}^{11}$ have compared refractive, topographic, pachymetric, tono- 
metric, and corneal biomechanical outcomes after ICRS insertion with or without prior CXL in 31 keratoconic eyes. They showed no statistical differences between groups postoperatively at 24 months for all parameters. However, the author's statement regarding keratometric and refractive outcomes being attributed entirely to CXL (after collagen cross-linking is performed) can be questioned in that ICRS are also contributing to the refractive and keratometric changes. Complete effect of ICRS may take more than 6 months to manifest. The immediate postoperative refractive outcomes are because of the ICRS segments in the periphery of the cornea resulting in the flattening of the central cornea and restoration of prolate anatomy. The European peer-reviewed keratoconus study and phases II and III myopia clinical trials noted that the refractive and keratometric changes to the cornea continue to manifest for more than 6 months postoperatively. ${ }^{12}$ In vivo confocal microscopy, ${ }^{13}$ and in vitro histological analysis ${ }^{14}$ of cadaveric/explanted animal and human corneal buttons have demonstrated that typical tissue response to ICRS insertion includes keratocyte activation (with resultant increase in keratocyte density), intracellular lipid accumulation, and new collagen formation manifesting as fibrosis around the Intacs segment. Although animal studies have reported that keratocyte activity in a healing cornea is greatest at 4 to 9 weeks and remains at a high level beyond 6 months, ${ }_{1}^{15}$ Ly et al's ${ }^{16}$ demonstration of absence of keratocyte activation or deposit formation at 2 months on confocal microscopy suggests that keratocyte activation in human eyes after ICRS implantation might occur after 2 months. The keratocyte activation and ensuing corneal remodeling explain the changes in refractive outcomes between 3 and 6 months. Additionally, the mid corneal layers continue to evolve and thicken over the time, which in turn may contribute to the biomechanical effects of Intacs. In the work by Ertan et al, ${ }^{9}$ the synergistic effect of CXL performed on average $3.98+/-5.7$ months after ICRS implantation might be explained in part by the continuing effect of ICRS-related corneal remodeling.

Combining both procedures in the same day is attractive for the patients and surgeons, reducing the time and cost of treatments, and probably minimizing the infectious risks. It remains unclear as to whether it is more effective to perform CXL before, during, or after ICRS insertion. If we postulate that ICRS can increase in efficacy over 6 months after insertion, ${ }^{12}$ it could be logical to wait 6 to 12 months before performing CXL. Similarly, does CXL performed prior ICRS implantation increase the mean corneal elasticity and lead to better biomechanical response after ICRS insertion? Performing both procedures on the same day is a reasonable choice, as no rational answer was provided to the above two questions in the literature. Devices based on transient elastography concept could potentially provide such answers in the near future. ${ }^{17}$ Nevertheless, the keratocyte apoptosis, when combining both procedures at the same time, could lead to a higher rate of corneal melting and extrusion. This theory is supported by a publication showing that the keratocyte density in the anterior part of the stroma above the rings, decreased dramatically after ICRS insertion. ${ }^{18}$ The same phenomenon was observed with CXL during the initial 6 months postoperatively, in the first anterior half of the cornea treated. ${ }^{19}$

Several studies have evaluated the rational for performing the ICRS implantation and collagen crosslinking the same day. In a prospective and comparative study, El-Raggal et al $^{20}$ evaluated the safety and efficacy of combined ICRS (Kerarings) insertion and CXL performed simultaneously or sequentially. They showed a statistically significant improvement in both groups in UDVA and CDVA, with a significant reduction in refractive error and keratometric values $(p<0.05)$ but no difference between both groups in all parameters. However, they reported an interesting finding in that stromal haze that developed in both groups was more marked and persistent after simultaneous treatment than after sequential treatment. Our group has recently conducted a retrospective study including 34 eyes of 34 patients with $\mathrm{KC}$ to evaluate the safety and efficacy of the simultaneous procedure; ICRS implantation and CXL the same day. ${ }^{21}$ Uncorrected visual acuity, BCVA, and SE manifest cylinder and keratometry $(\mathrm{K})$, were evaluated at baseline and at 1, 3, 6, and 12 months after surgery. After 6 months, the mean UCVA and BCVA improved by 3 lines. The minimum and maximum keratometry was reduced by a mean of 4 and $5 \mathrm{D}$ respectively and the mean SE decreased significantly with a mean of 2.3 D. One complication occurred at 1 month with ICRS extrusion through the corneal incision.

The combination of ICRS and CXL performed simultaneously seemed to be a minimally invasive treatment for patients with progressive $\mathrm{KC}$ and contact lens intolerance that sufficiently improves visual acuity and refraction. The visual acuity improvement reported by our group was similar to what was found by Coskunseven et al after a sequential treatment (ICRS implantation followed by CXL). ${ }^{10}$ However, regarding the lack of data in the literature, studies with longer follow-up are necessary to evaluate the stabilization over time and the clinical benefit of combining both treatments. Based on our recent finding, we presume that combining CXL and ICRS insertion in the same time seems to be safe and effective enough to be performed routinely. 


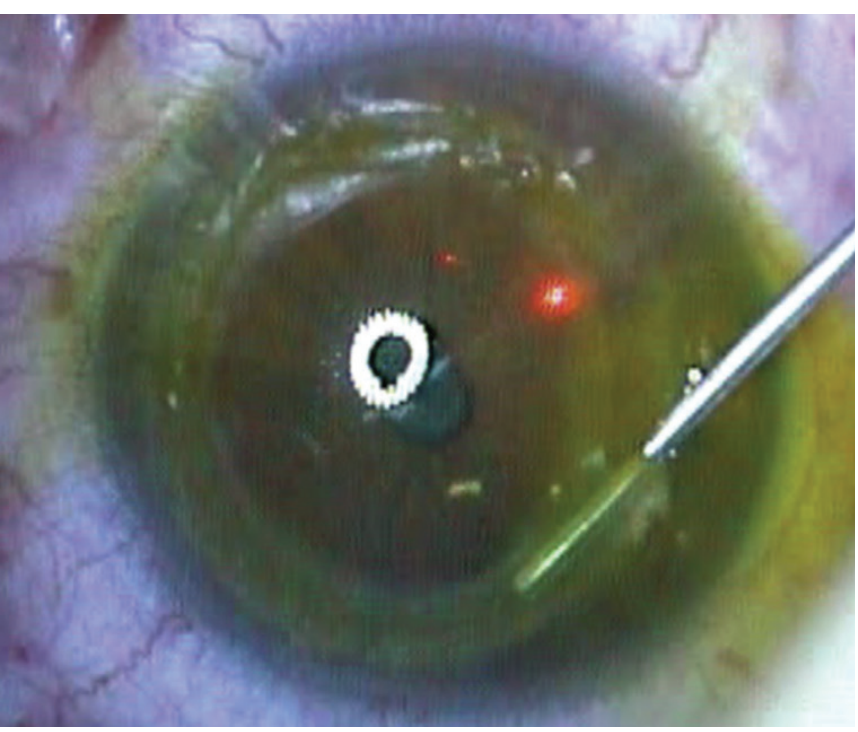

Fig. 2: Simultaneous transepithelial $C X L$ with riboflavin injection into the corneal channel

\section{SIMULTANEOUS TREATMENT WITH INTRACORNEAL RIBOFLAVIN INJECTION}

As an alternative to epithelial removal, Kanellopoulos introduced the technique of riboflavin administration directly into the stroma via a femtosecond laser-created intrastromal pocket. ${ }^{22}$ The thought process behind this alternative technique was that the creation of a femtosecond laser pocket with a small $10^{\circ}$ side cut would not weaken the cornea biomechanically, since it has been shown that the regular 270- to $290^{\circ}$ LASIK flap side cut may induce cornea weakening. ${ }^{23}$ Further, the selection of the exact depth that the riboflavin would be introduced could make the CXL procedure more customized instead of applying UV light and riboflavin to the whole cornea and potentially even at the level of the cornea endothelium.

Recently, Alio et al ${ }^{24}$ introduced a novel approach for combined ICRS implantation with CXL, where the intrastromal pocket created for ICRS implantationcreated with a femtosecond laser system at a corneal depth ranging from 70 to $90 \mu \mathrm{m}$ with a $70^{\circ}$ side cut and $7 \mathrm{~mm}$ diameter-was utilized for riboflavin administration. Riboflavin $0.1 \%$ in dextrose was injected directly into the corneal pocket. Significant corneal haze was observed in all cases in the early period but resolved over time. No postoperative pain reporting was observed in any case operated with the pocket technique. ${ }^{25}$ In this study, all patients enrolled had undergone previous corneal surgery, ICRS. A similar technique animal study was published by Dong and Zhou. ${ }^{7}$

Kıliç et al proposed a technique for riboflavin application without epithelial removal. ${ }^{26}$ In this technique, patients underwent combined CXL and ICRS and simultaneous transepithelial CXL with riboflavin injection into the corneal channel (Figs 2 and 3).

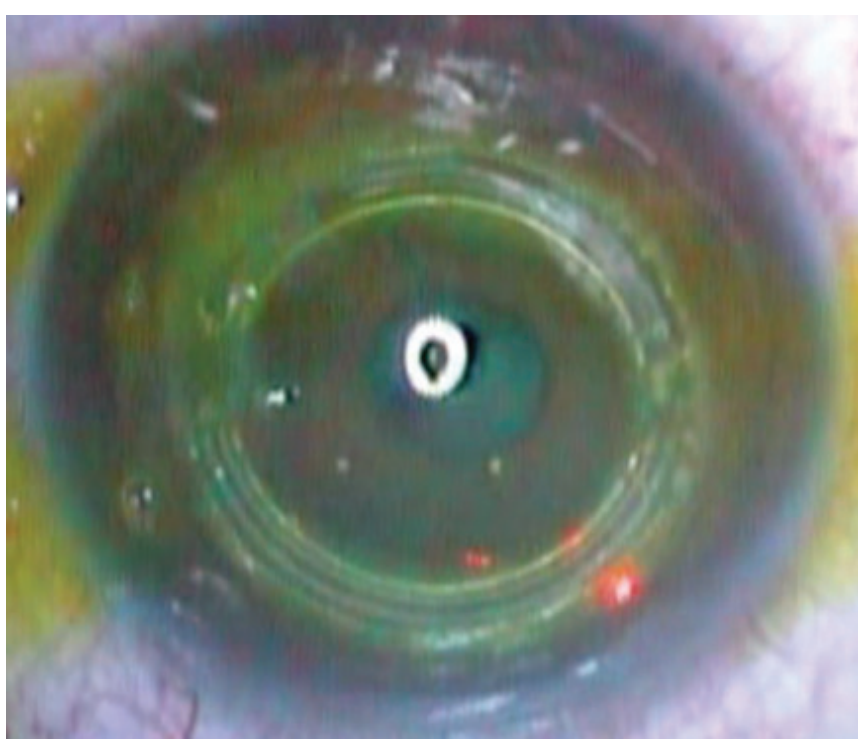

Fig. 3: After riboflavin injection, ICRS is implanted immediately

Riboflavin solution was injected with a 25 -gauge cannula into the corneal femto channel until riboflavin passed completely $360^{\circ}$ and filled all channel size. Then, immediately, Intacs segments were implanted inferiorly and superiorly based on patients' preoperative SE and the location of the cone.

\section{REFERENCES}

1. Barraquer JL. Refractive keratoplasty. Vol 1. Bogota, Columbia: Instituto Barraquer de America; 1970.

2. Colin J, Cochener B, Savary G, Malet F, Holmes-Higgin D. INTACS inserts for treating keratoconus: one-year results. Ophthalmology 2001 Aug;108(8):1409-1414.

3. Miranda D, Sartori M, Francesconi C, Alleman N, Ferrara P, Campos M. Ferrara intrastromal corneal ring segments for severe keratoconus. J Refract Surg 2003 Nov-Dec;19(6): 645-653.

4. Alió JL, Shabayek MH, Artola A. Intracorneal ring segments for keratoconus correction: long-term follow-up. J Cataract Refract Surg 2006 Jun;32(6):978-985.

5. Kymionis GD, Siganos CS, Tsiklis NS, Anastasakis A, Yoo SH, Pallikaris AI, Astyrakakis N, Pallikaris IG. Longterm follow-up of intacs in keratoconus. Am J Ophthalmol 2007 Feb;143(2):236-244.

6. Wollensak G, Spoerl E, Seiler T. Riboflavin/ultraviolet-ainduced collagen Crosslinking for the treatment of keratoconus. Am J Ophthalmol 2003 May;135(5):620-627.

7. Wollensak G, Spoerl E, Seiler T. Stress-strain measurements of human and porcine corneas after riboflavin-ultravioletA-induced cross-linking. J Cataract Refract Surg 2003 Sep;29(9):1780-1785.

8. Chan CC, Sharma M, Wachler BS. Effect of inferior-segment intacs with and without C3-R on keratoconus. J Cataract Refract Surg 2007 Jan;33(1):75-80.

9. Ertan A, Karacal H, Kamburo lu G. Refractive and topographic results of transepithelial cross-linking treatment in eye with intacs. Cornea 2009 Aug;28(7):719-723.

10. Coskunseven E, Jankov MR 2nd, Hafezi F, Atun S, Arslan E, Kimionis GD. Effect of treatment sequence in combined 
intrastromal corneal rings and corneal collagen crosslinking for keratoconus. J Cataract Refract Surg 2009 Dec;35(12): 2084-2091.

11. Renesto Ada C, Melo LA Jr, Sartori Mde F, Campos M. Sequential topical riboflavin with or without ultravioleta a radiation with delayed intracorneal ring segment insertion for keratoconus. Am J Ophthalmol 2012 May;153(5): 982-993.

12. Colin J. European clinical evaluation: use of intacs for the treatment of keratoconus. J Cataract Refract Surg 2006 May;32(5):747-755

13. Ruckhofer J, Böhnke M, Alzner E, Grabner G. Confocal microscopy after implantation of intrastromal corneal ring segments. Ophthalmology 2000 Dec;107(12):2144-2151.

14. Twa MD, Ruckhofer J, Kash RL, Costello M, Schanzlin DJ. Histologic evaluation of corneal stroma in rabbits after intrastromal corneal ring implantation. Cornea 2003 Mar;22(2): 146-152.

15. Katakami C, Sahori A, Kazusa R, Yamamoto M. Keratocyte activity in wound healing after epikeratophakia in rabbits. Invest Ophthalmol Vis Sci 1991;32(6):1837-45.

16. Ly LT, McCulley JP, Verity SM, Cavanagh HD, Bowman RW, Petroll WM. Evaluation of intrastromal lipid deposits after intacs implantation using in vivo confocal microscopy. Eye Contact Lens 2006 Jul;32(4):211-215.

17. Tanter M, Touboul D, Gennisson JL, Bercoff J, Fink M. Highresolution quantitative imaging of cornea elasticity using supersonic shear imaging. IEEE Trans Med Imaging. 2009 Dec;28(12):1881-1893.

18. Samimi S, Leger F, Touboul D, Colin J. Histopathological findings after intracorneal ring segment implantation in keratoconic human corneas. J Cataract Refract Surg. 2007 Feb;33(2):247-253.
19. Mazzotta C, Balestrazzi A, Traversi C, Baiocchi S, Caporossi $\mathrm{T}$, Tommasi C. Treatment of progressive keratoconus by riboflavin-UVA-induced cross-linking of corneal collagen: ultrastructural analysis by Heidelberg Retinal Tomograph II in vivo confocal microscopy in humans. Cornea 2007 May;26(4):390-397.

20. El-Raggal TM. Sequential versus concurrent KERARINGS insertion and corneal collagen cross-linking for keratoconus. Br J Ophthalmol 2011 Jan;95(1):37-41.

21. Touboul D. Simultaneous intra-corneal ring segments implantation and corneal collagen cross-linking for progressive keratoconus. Personnal communcation, XXVII Congress of the ESCRS, Barcelona; Sept 2009.

22. Kanellopoulos AJ. Corneal collagen crosslinking with intralase-assisted intracorneal riboflavin administration. Presented at: ISRS/AAO Annual Meeting; New Orleans: LA; November 10, 2007.

23. Knox Cartwright NE, Tyrer JR, Jaycock PD, Marshall J. Effects of variation in depth and side cut angulations in LASIK and thin-flap LASIK using a femtosecond laser: a biomechanical study. J Refract Surg. 2012 Jun;28(6):419-425.

24. Alió JL, Toffaha BT, Piñero DP, Klonowski P, Javaloy J. Cross- linking in progressive keratoconus using an epithelial debridement or intrastromal pocket technique after previous corneal ring segment implantation. J Refract Surg. 2011 Oct;27(10):737-743

25. Dong Z, Zhou X. Collagen cross-linking with riboflavin in a femtosecond laser-created pocket in rabbit corneas: 6-month results. Am J Ophthalmol. 2011 Jul;152(1):22-27.

26. Kılıç A, Kamburoglu G, Akıncı A. Riboflavin injection into the corneal channel for combined collagen crosslinking and intrastromal corneal ring segment implantation. J Cataract Refract Surg 2012 May;38(5):878-883. 